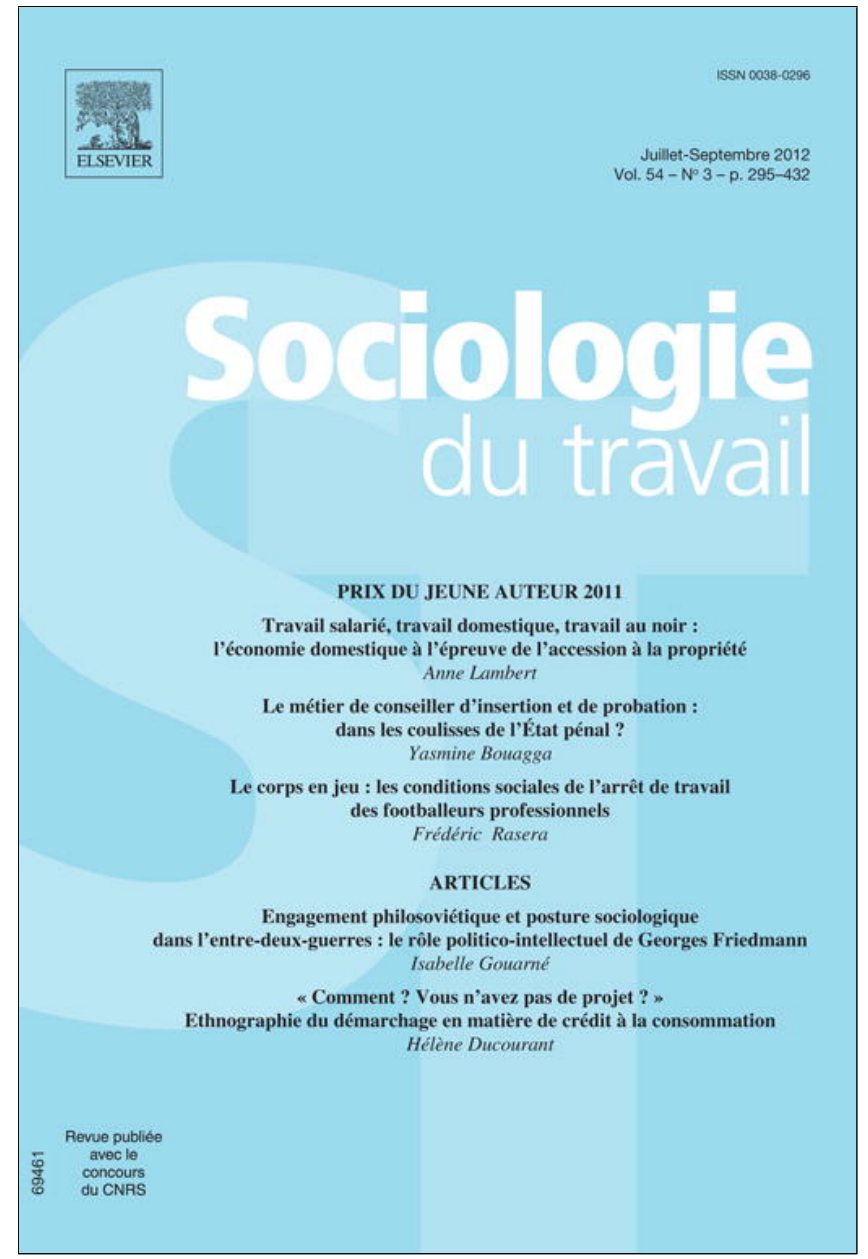

This article appeared in a journal published by Elsevier. The attached copy is furnished to the author for internal non-commercial research and education use, including for instruction at the authors institution and sharing with colleagues.

Other uses, including reproduction and distribution, or selling or licensing copies, or posting to personal, institutional or third party websites are prohibited.

In most cases authors are permitted to post their version of the article (e.g. in Word or Tex form) to their personal website or institutional repository. Authors requiring further information regarding Elsevier's archiving and manuscript policies are encouraged to visit:

http://www.elsevier.com/copyright 


\title{
Engagement philosoviétique et posture sociologique dans l'entre-deux-guerres : le rôle politico-intellectuel de Georges Friedmann
}

\author{
"Philosoviet" commitments and a sociological stance between the two \\ World wars: Georges Friedmann's political and intellectual role
}

Isabelle Gouarné*

Équipe Cultures et Sociétés urbaines, CNRS, centre de recherches sociologiques et politiques de Paris, université Paris 8, 59/61, rue Pouchet, 75849 Paris cedex 17, France

\section{Résumé}

Cet article propose une enquête biographique approfondie de la trajectoire de Georges Friedmann durant l'entre-deux-guerres. Réalisée à partir de ses archives privées et des archives du communisme, cette étude montre le rôle charnière qu'il a joué entre le monde communiste et les milieux universitaires français. Avec cette position, G. Friedmann a fortement contribué, dans les années 1930, à la légitimation du marxisme dans les sciences sociales françaises, même auprès des Durkheimiens qui s'étaient pourtant montrés si réfractaires aux idées de Karl Marx et Friedrich Engels depuis la fin du XIX ${ }^{\mathrm{e}}$ siècle. La posture politico-intellectuelle de G. Friedmann deviendra, cependant, intenable à la fin des années 1930, en raison de la fermeture du monde communiste.

(C) 2012 Elsevier Masson SAS. Tous droits réservés.

Mots clés : Histoire des sciences sociales ; Georges Friedmann ; Communisme ; Marxisme ; Étude du travail et des techniques

\section{Abstract}

This in-depth biographical study of Georges Friedmann's itinerary during the period between the two World wars draws from private records and the Communist movement's archives. It highlights this sociologist's pivotal role between the Communist world and academic circles in France. In the 1930s, Friedmann helped to legitimate Marxism in the French social sciences, even among Durkheimians who had proven resistant to Karl Marx's and Friedrich Engels's ideas since the end of the 19th century. Friedmann's

\footnotetext{
* Auteur corresondant.

Adresse e-mail : isabelle_gouarne@hotmail.com
} 
political and intellectual stance would, however, become untenable in the late 1930s given the closing of the Communist world.

(C) 2012 Elsevier Masson SAS. All rights reserved.

Keywords: History of social sciences; Georges Friedmann; Communism; Marxism; Study of work and techniques; France

La construction des traditions intellectuelles a été, depuis une vingtaine d'années, un objet privilégié de l'histoire sociale des sciences. En rupture avec les récits disciplinaires centrés sur les «pères fondateurs », cette approche invite à examiner les interprétations et les usages dont ont fait l'objet, à divers moments, les «précurseurs », les « classiques » et les « écoles » (Topalov, 2001). L'attention aux processus de reconstruction du passé a ainsi permis de revisiter l'histoire du durkheimisme et de ses rapports avec les autres pensées sociologiques. A été étudiée, en particulier, la réception que connaît en France Max Weber au cours des années 1960 et 1970, dans une période donc de remise en cause des traditions positivistes (Pollak, 1988). En revanche, alors que Karl Marx figure, encore aujourd'hui, aux côtés d'Émile Durkheim et de Max Weber, parmi les «pères fondateurs » de la discipline sociologique, les conditions d'introduction de ses idées n'ont guère été examinées.

L'émergence de la référence marxiste était pourtant loin d'aller de soi, ne serait-ce qu'en raison des réticences, maintes fois répétées, de la première et deuxième générations durkheimiennes vis-à-vis de cette pensée. Les critiques formulées par E. Durkheim en 1897 contre le matérialisme historique (Lacroix, 1978) ${ }^{1}$ furent, en effet, largement reprises durant l'entre-deux-guerres par les Durkheimiens, tels Célestin Bouglé ou Maurice Halbwachs, qui continuaient à opposer radicalement sociologie et marxisme et qui, en même temps, affichaient leur opposition à la Révolution bolchevique (Humphreys, 1999; Verret, 1972). De fait, on ne peut étudier le marxisme au $\mathrm{XX}^{\mathrm{e}}$ siècle, sans tenir compte de ce qui fut une des spécificités de ce courant, celle de s'imposer dans les institutions universitaires, alors que l'étiquette marxiste était déjà revendiquée par des mouvements politiques et, à partir de 1917, par l'État soviétique. Comprendre comment les idées de Karl Marx et Friedrich Engels sont devenues des références légitimes dans l'univers académique français suppose donc d'articuler l'histoire des sciences humaines à une histoire sociale des idées politiques, et c'est en suivant cette orientation de recherche qu' on se propose de revenir sur l'itinéraire de Georges Friedmann (1902-1979), qui compte parmi les premiers sociologues français à acquérir, au cours des années 1930, une position académique tout en se revendiquant de la pensée de K. Marx et F. Engels.

Georges Friedmann, bien plus, est parvenu, dans l'entre-deux-guerres, à inventer et à faire exister un nouveau rôle d'intellectuel, associant production sociologique et engagement philosoviétique ${ }^{2}$. C'est ce qui ressort d'une enquête biographique réalisée à partir de ses archives privées $^{3}$ et des archives politiques du communisme, conservées en Russie et en France ${ }^{4}$. Ces

\footnotetext{
${ }^{1}$ Cf. la recension qu'É. Durkheim livre, en 1897, dans la Revue philosophique sur l'ouvrage d'A. Labriola (repris dans Durkheim, 1987).

${ }^{2}$ Sur le mode d'analyse de ce type d'itinéraire, voir l'étude de cas proposée par B. Pudal sur Paul Nizan, condisciple de G. Friedmann à l'École normale supérieure et lui aussi engagé, au cours des années 1930, dans une double carrière, politique au Parti communiste et intellectuel-littéraire. Cf. (Pudal, 2002).

3 Je tiens ici à remercier vivement Liliane Boccon-Gibod qui, avec une grande disponibilité, m'a permis de consulter les archives de son père.

${ }^{4}$ Cette recherche biographique s'appuie notamment sur les archives du communisme conservées à Moscou, celles de l'Internationale communiste, notamment de la Section Éditions (Archives d'histoire sociale et politique de l'État de
} 
recherches permettent d'éclairer les homologies et les rapports, déjà mis au jour, entre ses prises de position politiques et sa production savante (notamment, Ribeill, 1999; Rot et Vatin, 2004 ; Vatin, 2004 ; Grémion et Piotet, 2004), en révélant le rôle charnière qu'il a joué entre le monde communiste et les milieux universitaires français. Bien que contrainte par cette double insertion, la production intellectuelle de G. Friedmann a bénéficié d'une forte reconnaissance dans l'univers des sciences sociales françaises, de plus en plus politisées à partir de 1934, et a ainsi favorisé la légitimation académique du marxisme. Sa posture politico-intellectuelle deviendra toutefois intenable à la fin des années 1930, en raison de la fermeture du monde communiste.

\section{L'invention d'un nouveau rôle intellectuel ou comment être sociologue et communiste dans l'entre-deux-guerres}

Né en 1902, G. Friedmann compte parmi les intellectuels qui, issus des jeunes générations d'après-guerre, eurent à s'intégrer dans une société profondément bouleversée. C'est dans ce contexte, marqué par le rejet de la Première Guerre mondiale et des élites qui l'ont rendue possible, que G. Friedmann rompt avec son histoire sociale familiale et la destinée que son père, banquier, lui avait tracée (la haute finance ou l'industrie), en s'orientant vers une carrière intellectuelle et en s'engageant aux côtés du mouvement communiste ${ }^{5}$. Son rapport au communisme soviétique s'enracine ainsi dans un double mouvement : un mouvement de contestation tous azimuts de l'ordre établi et des élites traditionnelles ; un mouvement de projection vers l'URSS, bien symbolique, objet de multiples appropriations, où il est possible d'investir sa quête d'un nouvel ordre social (Pudal, 1989, pp. 30-37).

Dès les années 1920, alors que G. Friedmann poursuit sa scolarité à l'École normale supérieure (ENS) de la rue d'Ulm, son philosoviétisme se manifeste par des positions avant-gardistes associant subversion culturelle et radicalité politique. En témoigne sa participation à la revue Clarté (Racine, 1967) ainsi qu'aux activités du groupe Philosophies (Trebitsch, 1987). Toutefois, l'ouvriérisme et l'anti-intellectualisme qui marquent alors le Parti communiste (PC), notamment durant la seconde moitié des années 1920, rendent difficiles ces positionnements. Les pratiques militantes de G. Friedmann resteront de ce fait cantonnées aux marges de l'espace partisan, et ce jusqu' au début des années 1930. À cette époque, les transformations du monde communiste offrent aux intellectuels de profession de nouvelles possibilités militantes que G. Friedmann sut saisir pour s'aménager une position originale, située à la jonction de l'univers académique et du monde politique. Cette position implique, on le verra, trois rôles : un rôle de passeur dans les circulations scientifiques franco-soviétiques, un rôle d'entrepreneur culturel et un rôle de sociologue.

\subsection{Un passeur dans les circulations scientifiques franco-soviétiques}

À partir de 1932, lorsque les échanges entre la France et l'URSS reprennent et que l'action de la diplomatie culturelle soviétique se déploie (Coeuré, 1999; Mazuy, 2002; Rey, 2002), G. Friedmann réussit à s'imposer comme un intermédiaire entre les deux pays: son

Russie, RGASPI), et celles de la diplomatie culturelle soviétique (archives du VOKS, l'organisation chargée des relations culturelles avec l'étranger, conservées aux Archives gouvernementales de Russie, GARF). En France, ont notamment été consultées les archives Maurice Thorez (Archives nationales).

${ }^{5}$ Cette problématique est d'ailleurs au cœur de son premier roman, Votre tour viendra (Gallimard, 1930), dans lequel il entend examiner, «à travers tout le chaos de l'après-guerre », « les problèmes de [sa] génération ». 
philosoviétisme affiché et son action militante lui permettent alors de nouer en URSS un réseau de relations personnelles.

Début 1932, en effet, G. Friedmann s'engage activement au sein du Cercle de la Russie neuve. Cette association d'intellectuels fut lancée en 1927 par le Vsesojuznoe obščestvo kulturnoj svjazi s zagranicej (VOKS) (Coeuré, 2012; David-Fox, 2012; Fayet, 2003) : l'organisation soviétique chargée des échanges culturels avec l'étranger visait par là à mieux faire connaître les « réalisations soviétiques » dans les milieux intellectuels français et à y créer un mouvement de sympathie à l'égard de l'URSS. À ses débuts, le Cercle de la Russie neuve se consacre pour l'essentiel à la diffusion de films soviétiques, et ses activités restent peu intenses. En 1932, cependant, le VOKS se montre soucieux de redynamiser cette association et décide alors de la restructurer en plusieurs commissions (notamment, cinématographique, théâtrale, économique et scientifique) ${ }^{6}$. Il entend, de plus, renforcer son action à l'étranger en nouant des liens individuels « amicaux » avec des intellectuels soigneusement choisis (Stern, 2007) — non seulement avec des personnalités reconnues, mais aussi avec de jeunes intellectuels aux positions académiques moins assurées mais disposant de temps et d'énergie. Ce fut notamment le cas de G. Friedmann qui tissa, à cette époque, des liens personnels étroits avec des responsables du $\mathrm{VOKS}^{7}$ et qui, de fait, aux yeux des Soviétiques, présentait toutes les qualités requises.

Ancien élève de l'ENS de la rue d'Ulm et agrégé de philosophie (1926), il est doté de diplômes socialement valorisés. Il occupe, en outre, depuis 1931, la fonction de secrétaire-archiviste au Centre de documentation sociale que dirige, à l'ENS, Célestin Bouglé et qui constitue, à cette époque, un lieu de sociabilités intellectuelles important. Ce poste — assez recherché du reste, puisqu'il permet d'éviter une affectation dans un lycée de province, renforce la capacité de mobilisation politique de G. Friedmann. Il saura d'ailleurs en jouer, début 1932, lors du lancement de la commission scientifique du Cercle de la Russie neuve, en recrutant à l'ENS de jeunes intellectuels (Jean Luc, Henri Mougin, Jacques Soustelle, par exemple). Recommandé auprès du VOKS par Henri Barbusse, il réalise son premier séjour en URSS en septembre-octobre 1932. La commission scientifique du Cercle de la Russie neuve, présidée par le psychologue Henri Wallon, le charge alors d'établir « la liaison entre les travailleurs intellectuels de différentes spécialités qui se sont groupés dans cette commission et les institutions ou personnalités scientifiques de l'URSS $»^{8}$. G. Friedmann, qui apprend le russe, deviendra peu de temps après le secrétaire de cette commission scientifique. Il sera ainsi un interlocuteur privilégié du VOKS, pour lequel il joue un rôle d'informateur sur la situation économique, politique et idéologique du monde intellectuel français ${ }^{9}$. En retour, le VOKS l'accueille avec égards lors de ses voyages successifs en URSS, en 1932, puis en 1933 et 1936 (Mazuy, 2004), il l'appuie dans ses recherches sur l'organisation du

\footnotetext{
6 Sur les activités du Cercle de la Russie neuve, cf. les archives du VOKS, notamment F. 5283, Op. 7, D. 56, D. 109, D. 112, D. 163, D. 254, D. 407, D. 455 (GARF). Cf. (Gouarné, 2010).

7 Voir la correspondance de G. Friedmann avec les rédacteurs en chef de la revue Littérature internationale (C.C. Dinamov et T.A. Rokotov) et avec M. J. Apletin (secrétaire de la Commission extérieure de l'Union des écrivains soviétiques et vice-Président du VOKS) reproduite dans T. Balachova (ed.), Dialog pisatelej. Iz istorii russko-francuzskih kulturnyh svjazej XX veka, 1920-1970, Moscou, Ed. IMLI RAN, 2002 (Dialogues d'écrivains. Pages d'histoire des relations culturelles franco-russes au $\mathrm{XX}^{e}$ siècle, 1920-1970).

${ }^{8}$ Lettre du Président de la Commission scientifique du Cercle de la Russie neuve à Rotman, Chef de la section latine du VOKS, 14 juillet 1932. Fonds Lahy, F. 275, Op. 1, D. 749 (Musée historique de Sainte Anne).

${ }^{9}$ Lors de son second voyage en URSS (septembre-octobre 1933) notamment, il prononça le 9 octobre au VOKS une conférence sur la situation des intellectuels en France (F. 5283, Op. 8, D. 201, L. 85-101, GARF). En général, le VOKS profite de la venue en URSS d'intellectuels étrangers pour s'informer sur les diverses situations nationales.
} 
travail en pays socialiste et il facilite ses contacts — en même temps qu'il les contrôle ${ }^{10}$ — dans les milieux intellectuels soviétiques.

En raison de ses séjours répétés en Russie et des enquêtes sociologiques qu'il put y mener grâce à sa maîtrise du russe et à ses liens politiques, G. Friedmann apparaît donc dans l'univers des sciences sociales françaises des années 1930 comme un des principaux intermédiaires avec l'URSS.

\subsection{Un entrepreneur culturel dans l'univers communiste}

En France, la redéfinition de la politique culturelle du PC, à partir de 1932 et surtout au moment du Front populaire, ouvre de nouvelles perspectives aux intellectuels de profession (Pudal, 2004 ; Matonti, 2004). Les obstacles politiques qui, dans ce milieu partisan, leur interdisaient toute fonction à responsabilité sont momentanément levés. Bien qu'il ne soit pas adhérent communiste, G. Friedmann put alors endosser un rôle d'entrepreneur culturel.

En 1935, notamment, il fut recruté aux Éditions sociales internationales (ESI), une des principales maisons d'édition du PC, comme directeur de la nouvelle collection « Socialisme et culture ». La visée de cette dernière était le «recensement de toutes les forces humanistes » dont la pensée de K. Marx et F. Engels était posée comme héritière ; un volume devait ainsi être consacré à chaque penseur du «grand courant humaniste et socialiste» que prétendait désormais incarner le PC. Le lancement de cette collection fut aussi une des manifestations concrètes de sa politique d'ouverture aux intellectuels : elle a créé un espace éditorial leur permettant de contribuer à l'entreprise culturelle communiste.

On connaît mal le processus de décision qui conduit à la nomination de G. Friedmann hormis le soutien dont il bénéficie de la part de Léon Moussinac qui, depuis 1935, assurait, avec René Hilsum, la direction des ESI (Bouju, 2010). Il est clair, cependant, que la force de mobilisation qu'il détient, en raison de sa trajectoire scolaire et de sa position au Centre de documentation sociale de l'ENS, lui confère une autorité spécifique dans le monde communiste. Dans un rapport daté de 1935, relatif à des projets de publications aux ESI, le choix de G. Friedmann comme directeur de la collection « Socialisme et culture» est justifié, en effet, par les « relations personnelles de cet écrivain et l'autorité autant que l'estime dont il jouit dans les milieux littéraires et scientifiques et spécialement dans l'Université ${ }^{11}$. En outre, dans l'espace politique communiste où les liens personnels fonctionnent comme caution morale et politique, G. Friedmann peut se prévaloir de relations avec des responsables soviétiques, ceux du VOKS on l'a vu, et aussi avec des intellectuels communistes proches désormais du cercle dirigeant thorézien, tels Georges Cogniot et Paul Nizan ${ }^{12}$ qu'il connaît depuis sa scolarité à l'ENS. Sa position de « sympathisant » (et non de membre du PC), souvent analysée comme la marque d'une distance recherchée à l'égard de ce Parti, lui permet en réalité d'accroître sa capacité de mobilisation parmi les intellectuels. Dans les milieux universitaires, au sein desquels le PC cherche maintenant à étendre son influence, sans pour autant désirer des adhésions, G. Friedmann constitue un relais d'autant plus opérant qu'il n'y apparaît pas comme mandaté par la direction partisane et qu'il peut afficher une certaine autonomie.

\footnotetext{
${ }^{10}$ En 1936, le refus de G. Friedmann d'être accompagné par le responsable du VOKS lors d'une rencontre avec le psychologue soviétique Šidlovsk qu'il connaissait déjà fera ainsi l'objet d'une note critique de son guide (Coeuré et Mazuy, 2012, document 101).

${ }^{11}$ Note de L. Moussinac, 7 août 1935. Annexe 3 (suite). Projet de publication sur la guerre et le fascisme. Fonds de l'Internationale communiste, Section Éditions : F. 495, Op. 78, D. 135 (RGASPI).

12 Voir leurs notices biographiques dans (Pennetier, 1997).
} 
En tant que directeur de collection, G. Friedmann a pour fonction, en premier lieu, de recruter des auteurs, afin de renforcer l'autorité intellectuelle des ESI. De fait, grâce à lui, cette maison d'édition du PC s'est ouverte aux milieux universitaires: aux côtés d'ouvrages d'intellectuels communistes ou sympathisants (Jean Cassou, Valentin Feldman, René Maublanc, Paul Nizan, Marcel Prenant), la collection « Socialisme et culture » fera paraître, en 1938, un ouvrage du germaniste Edmond Vermeil sur Henri Heine, et elle pourra aussi annoncer deux livres, jamais édités en fait, de l'historien Lucien Febvre sur les Humanistes de la Renaissance. Le rôle de G. Friedmann comprenait également un volet politique, comme le notait à l'époque Léon Moussinac : «il y aura de nombreuses et délicates révisions de textes à faire, tant plus qu'on s'adressera, comme il est nécessaire, à des écrivains encore éloignés de nous malgré leur nette position antifasciste ${ }^{13}$. En assurant la fonction de directeur de la collection « Socialisme et culture », G. Friedmann devenait donc un «homme double (Charle, 1998, pp. 89-95), un médiateur, pris entre des logiques et des exigences éditoriales, politiques et intellectuelles.

On peut saisir les difficultés que soulevait une telle position en lisant la correspondance que G. Friedmann a échangée avec Alexandre Kojève, ce philosophe originaire de Moscou, qui, après son départ de la Russie en 1920, s'était installé d'abord en Allemagne, puis, à partir de 1926, à Paris. Avec ce parcours migratoire, A. Kojève s'est imposé, on le sait, comme un des principaux introducteurs de la pensée de Hegel en France (Jarczyk et Labarrière, 1990). On n'a, en revanche, guère souligné comment, au milieu des années 1930, alors qu'il tentait de concilier sa lecture de Hegel avec une approche marxiste, A. Kojève se rapprocha des milieux intellectuels philosoviétiques. En 1937 notamment, il envisagea de publier aux ESI, dans la collection «Socialisme et culture», un ouvrage sur Bernard de Fontenelle et Pierre Bayle, auquel d'ailleurs il consacrait alors la moitié de son cours à l'EPHE (Filoni, 2010, pp. 240-251). Le synopsis détaillé qu'il rédigea en juillet 1937, bien qu'il fut jugé par G. Friedmann «très intéressant et fouillé », suscita quelques réserves - de forme d'abord, A. Kojève étant invité à adopter le style «le plus clair et le plus vivant» possible, mais aussi de fond ${ }^{14}$. En effet, si son étude soulevait des enjeux politiques forts (en particulier, la question de la légitimité de l'État à intervenir dans le domaine des sciences), il ne répondait pas totalement aux attentes de la collection « Socialisme et culture », dont la principale visée était de construire de nouvelles généalogies politiques et intellectuelles et par là de renforcer la légitimité du PC : étudier le «rôle historique » de tel ou tel penseur, en le situant «dans le courant d'idées qui va, à travers la critique rationaliste, vers l'Encyclopédie, la critique de l'Église et de la religion chez les Philosophes, vers la Révolution française », analyser «l'influence réelle de ces courants» et «dégager les grandes lignes des mouvements d'idées qui ont permis la formation de la pensée socialiste ${ }^{15}$, voilà ce à quoi G. Friedmann tentait d'amener A. Kojève et tous les auteurs qu'il sollicitait pour sa collection aux ESI.

\subsection{Un sociologue dans le monde communiste}

Enfin, la position politico-intellectuelle occupée par G. Friedmann impliquait une articulation étroite entre production savante et engagement politique. Son recours à la notion de machinisme

\footnotetext{
13 Note de L. Moussinac, 7 août 1935. Annexe 3 (suite). Projet de publication sur la guerre et le fascisme. Fonds de l'Internationale Communiste, Section Éditions : F. 495, Op. 78, D. 135 (RGASPI).

${ }^{14}$ Cf. Lettre de G. Friedmann à A. Kojève, 18 juillet 1937. Fonds A. Kojève (BNF, Département des Manuscrits, Boîte 20).

15 Ibidem.
} 
est à cet égard symptomatique, puisque, comme le note François Vatin, ce concept présente, dans l'entre-deux-guerres, une double légitimité, politique et scientifique (Vatin, 2004) ${ }^{16}$. Les lieux de publication de ses écrits témoignent également de son désir de concilier production sociologique et militantisme communiste: alors que son livre Problèmes du machinisme en URSS et dans les pays capitalistes paraît aux ESI en 1934, c'est chez Gallimard qu'il publie, en 1936, la Crise du progrès, ouvrage qui devait du reste être traduit en russe ${ }^{17}$.

Ces deux ouvrages, malgré des modes de présentation différents, reposent sur la même structure argumentative: (1) analyse socio-historique du développement du capitalisme; (2) examen des deux principaux systèmes d'organisation du travail industriel, ceux de Ford et de Taylor : critiques de ces idéologies, à partir notamment des travaux psychotechniques français (J.-M. Lahy, H. Wallon, notamment) et soviétiques (I.N. Spielrein, J. Ermanski et al.); (3) définition, à partir de l'exemple soviétique, d'une «rationalisation socialiste et humaniste» (Ribeill, 1999; Vatin, 2004). Le communisme soviétique est donc considéré dans ces écrits comme le lieu où sont en passe d'être résolus les conflits entre l'homme et la technique, comme la solution aux impasses de la civilisation industrielle moderne. Se tourner vers l'URSS est ici un moyen d'envisager le progrès et le futur, de se démarquer des discours décadentistes, tout en restant soucieux du monde en voie de disparition. Ainsi, pour G. Friedmann, le progrès est possible à deux conditions. Une condition politique, d'abord : le progrès suppose la construction d'une société où la technique est mise au service de l'homme, permettant ainsi la libération de temps de loisirs et l'allégement de la charge du travail. La seconde condition, qui renvoie au rôle social de la science, est la nécessité de contrôler la machine par «la collaboration fraternelle des savants et des ingénieurs avec les ouvriers » : le progrès exige un « effort humaniste de la science pour protéger l'ouvrier contre les dangers de la machine» (Friedmann, 1935).

Ces analyses, on le voit, sont conformes aux positions adoptées alors dans le monde communiste sur l'organisation scientifique du travail. À partir de 1928, en effet, l'Internationale communiste, le PC et la CGTU opposent à la « rationalisation capitaliste » la «rationalisation socialiste », la seule qui permettrait, selon leurs termes, «la pleine utilisation au profit des travailleurs du rendement des conquêtes techniques de la science» (cité par Ribeill, 1984). Les écrits de G. Friedmann ne correspondaient pas cependant à un simple alignement du registre savant sur le registre politique. Ils supposaient aussi l'exercice de fonctions savantes d'expertise, la compréhension des mécanismes sociaux devant, aux yeux de G. Friedmann, servir de base à l'action politique rationnelle et cohérente. Après la Seconde Guerre mondiale, lorsque G. Friedmann ne sera plus engagé dans le mouvement communiste, cette posture sera clairement revendiquée dans ses écrits. Néanmoins, dès les années 1930, cette conception est sous-jacente à ses interventions politico-intellectuelles, même si elle est alors indicible au sein du PC, qui se stabilise à cette époque avec la promotion d'une élite ouvrière militante, soucieuse d'empêcher la réapparition de toute forme de contestation intellectuelle (Pudal, 1989, pp. 180-192).

En fait, les travaux de G. Friedmann sur l'organisation scientifique du travail et la notion de progrès participaient, dans les années 1930, d'un programme de recherches marxistes, centré sur

\footnotetext{
${ }^{16}$ La notion de «machinisme» est autorisée dans l'espace communiste, puisque dans la version française du Capital, parue dans les années 1870, le terme allemand de Maschinerie avait été traduit, avec l'accord de K. Marx, par «machinisme». Au sein de l'univers académique, cette notion est également légitime dans le domaine de l'histoire économique, où elle fut notamment discutée, en 1906, par Paul Mantoux dans sa thèse sur la Révolution industrielle au XVIII $^{\mathrm{e}}$ siècle (Vatin, 2004).

17 Cf. Lettre de G. Friedmann à C.C. Dinamov, 17 février 1937, dans Dialog pisatelej, op. cit., pp. 310. Ce projet ne put aboutir.
} 
l'histoire des techniques et articulé au projet de transformations sociales et politiques dont le PC était porteur. Ce programme avait émergé au sein du réseau d'intellectuels philosoviétiques, qui s'était formé, au début des années 1930, suite au travail de mobilisation politique réalisé par les instances communistes. La commission scientifique du Cercle de la Russie neuve, créée, rappelons-le, en 1932 sous l'impulsion de la diplomatie culturelle soviétique, s'était rapidement transformée en véritable «collectif de pensée» (Fleck, 2005 [1935]), caractérisé par un «style de pensée »étiqueté «marxiste ${ }^{18}$ et par un programme de recherches, dont l'hypothèse centrale était la suivante : le substrat social fondamental n'est pas, comme le pensait É. Durkheim, la morphologie sociale, mais le travail et les techniques, c'est-à-dire l'ensemble des activités humaines de production et de transformation de la nature. Dans la seconde moitié des années 1930, se rattachaient à ce programme de recherches, outre les travaux de G. Friedmann, ceux de Jean-Maurice Lahy en psychologie appliquée, ceux d'André-Georges Haudricourt et de Charles Parain sur l'histoire de l'outillage agricole ou encore ceux d'André Varagnac sur le folklore. Ces intellectuels, tous membres actifs de la commission scientifique du Cercle de la Russie neuve, tentaient alors de promouvoir une science sociale unifiée, fondée sur un postulat commun, définissant le travail comme étant le lien social par excellence.

\section{Les raisons intellectuelles et politiques d'une reconnaissance académique}

En associant ces trois rôles - de médiation scientifique franco-soviétique, d'entrepreneur culturel et de sociologue - , G. Friedmann fut sans doute, au cours des années 1930, l'intellectuel qui combina le plus étroitement carrière militante communiste et carrière universitaire. Et paradoxalement, cette façon d'articuler science et politique a été une des raisons de la reconnaissance académique de G. Friedmann : on le verra en étudiant les modalités de son insertion dans l'univers des sciences humaines françaises.

\subsection{Le Centre de documentation sociale, un espace académique ouvert aux jeunes intellectuels politisés}

C'est d'abord grâce à Célestin Bouglé ${ }^{19}$ et aux moyens dont il disposait au Centre de documentation sociale (CDS) que G. Friedmann put trouver, au début des années 1930, les ressources matérielles nécessaires au lancement de ses recherches et de sa carrière universitaire ${ }^{20}$. Fondé en novembre 1920, le CDS fut installé dans les locaux de l'ENS, rue d'Ulm. Il fonctionne jusqu'à la guerre sur les fonds alloués par le banquier A. Kahn (Coeuré, 1995), auxquels s'ajoutent en 1932 des financements de la Fondation Rockefeller (Tournès, 2008). Doté à l'origine d'une mission documentaire, le CDS deviendra, dans les années 1930, le principal lieu académique de formation à la sociologie en France (Marcel, 2001).

Célestin Bouglé, on le sait, a fait preuve d'une certaine tolérance, en recrutant au CDS de jeunes intellectuels non seulement militants, mais aussi très critiques vis-à-vis du durkheimisme,

\footnotetext{
18 Ce groupe fut notamment à l'origine de deux ouvrages collectifs intitulés À la lumière du marxisme et parus en 1935 et 1937 aux ESI.

19 Célestin Bouglé (1870-1940) fut, dès les années 1890, intégré à l'équipe d'É. Durkheim et connut rapidement une brillante carrière universitaire : en 1919, il fut nommé titulaire de la chaire d'histoire d'économie sociale à la Sorbonne et il fut également directeur adjoint (1927-1935), puis directeur de l'ENS (1935-1939).

20 Après avoir été assistant au CDS (1931-1934), il sera professeur à l'École Boulle (1935-1939) puis, après la Seconde Guerre mondiale, Professeur au CNAM (1946-1960), à l'IEP de Paris (1949-1960) et, à partir de 1960, à l'EPHE.
} 
qu'ils percevaient comme «un des symboles de ce qui était rejeté comme démodé et corrompu, en particulier le couplage d'une orientation positiviste et de la morale laïque » (Heilbron, 1985, pp. 229). Cette tolérance s'explique en partie par la position d'homme double que C. Bouglé a lui-même occupée, en restant, durant toute sa carrière académique, un radical-socialiste activement engagé (Logue, 1979; Dreyfus, 1997 ; Prochasson, 1999). Elle tient aussi aux difficultés de recrutement auxquelles les Durkheimiens étaient alors confrontés. Soucieux d'assurer la relève, C. Bouglé a mis en œuvre au CDS une politique d'ouverture intellectuelle et politique du paradigme durkheimien (Marcel, 2001). Ainsi, alors qu'il réaffirma à plusieurs reprises son opposition au projet bolchevique ${ }^{21}$ et son rejet du matérialisme historique ${ }^{22}$, il accueillit au CDS de jeunes intellectuels qui se revendiquaient du marxisme et affichaient leur philosoviétisme (G. Friedmann, mais aussi Auguste Cornu, Valentin Feldman, Henri Mougin, André Varagnac). En raison de cette ouverture, la «Docu» a donné l'image d'une science sociale progressiste, où, selon Le Figaro, «les principes révérés sont sinon rouges, du moins roses» (Le Figaro, 3 juin 1939, cité par S. Israël et J.-P. Mochon, 1994, pp. 199).

\subsection{Georges Friedmann aux Annales}

Plus étonnant peut-être est le soutien académique dont bénéficiera G. Friedmann de la part des deux directeurs des Annales. Il sera, dans la seconde moitié des années 1930, un collaborateur de la revue, avant d'entrer, en 1939, dans son comité de rédaction et de devenir, après la Seconde Guerre mondiale, un de ses directeurs. Pourtant, le militantisme de G. Friedmann n'apparaît guère conforme à la « déontologie du désengagement » défendue par M. Bloch et L. Febvre après la Première Guerre mondiale (Dumoulin, 2003, pp. 222-224). De fait, les deux directeurs des Annales feront preuve initialement d'une certaine réserve vis-à-vis de G. Friedmann, critiquant à plusieurs reprises son rapport ecclésial au marxisme. L. Febvre, par exemple, dans sa lettre du 2 juillet 1933 à M. Bloch, le présentera de la façon suivante :

«Je crois que nous aurons un technicien bientôt. J'ai vu deux ou trois fois ces temps-ci un jeune philosophe nommé Friedmann. Assez drôle de corps. Fils d'un banquier m'a-t-on dit, et qui a abjuré le capitalisme et ses rentes, a voulu vivre $d a$ se, a été touché par la grâce soviétique et par l'Évangile marxiste : c'est même très amusant de voir les yeux de ce gros garçon (extrêmement fin et intelligent, du reste) se mouiller quand on prononce le nom de Dieu et commencer ses discours par: «Comme le dit Marx, dans la partie II du Capital, au début du sixième chapitre...» ou encore: «S'ils avaient lu Marx, et lu : je veux dire compris (formule astucieusement ingénue qui permet la "mise à jour" continuelle dudit Marx), ils sauraient que...». À part ça, grosse thèse en train sur les problèmes à la fois sociologiques et psychologiques que posent l'organisation du travail, le taylorisme, etc. [...] On l'essaiera, car je ne sais pas comment il écrit, bien, me dit-on. En tout cas, il est intelligent et, foi à part, sait ce qu'il dit» (Bloch et Febvre, 1994, pp. 391-392).

\footnotetext{
${ }^{21}$ C. Bouglé est l'auteur en 1918 d'une brochure intitulée «Qu'est-ce que le bolchevisme», dans laquelle il accuse le bolchevisme d'être le «pire ennemi du socialisme » : «Le bolchevisme, écrit-il, est une expérimentation cruelle tentée sur le grand corps pantelant de la Russie par une équipe de docteurs sans scrupules ».

22 En mars 1935, par exemple, il consacrera une série de cours à la Sorbonne aux rapports entre marxisme et sociologie. Cet enseignement sera marqué par une double exigence : discuter les thèses marxistes du point de vue de «la sociologie », c'est-à-dire du point de vue durkheimien, et, en même temps, montrer tout ce qui sépare ces deux systèmes de pensée. Voir le résumé de son cours «Sociologie et marxisme » dans le Bulletin du groupe d'études philosophiques de la Faculté des lettres de Paris, 11, 14 mars 1935.
} 
En 1935, également, à propos de la contribution de G. Friedmann au numéro spécial des Annales consacré à l'histoire des techniques, M. Bloch met en garde L. Febvre : « Ayez l'autorité nécessaire pour lui rappeler en cas de besoin que le dogme est à sa place dans un bulletin paroissial, moins aux Annales » (Lettre du 8 octobre 1935, Bloch et Febvre, 2003, pp. 305).

Après 1935, ces réticences s'atténueront et, chez L. Febvre du moins, laisseront place à un véritable engouement pour le «marxisme vivant » mis en œuvre par G. Friedmann et d'autres. En mars 1936, notamment, il présidera une conférence publique organisée par le Cercle de la Russie neuve, dont, on l'a vu, G. Friedmann était un membre très actif, et en rendit compte de la façon suivante à M. Bloch :

«Les oreilles ont dû vous corner samedi soir. Je présidais pour la deuxième fois une réunion publique consacrée à la discussion d'À la lumière du marxisme. Exposé remarquable de Friedmann, entre autres, lequel s'appuyant sur vous et sur Sion, a fort bien expliqué à son peuple que Lefebvre des Noëttes, ce n'était pas le marxisme — loin de là — et que mettre d'un côté l'esclavage, de l'autre l'attelage, établir le contact et puis dire : volatilisé, l'esclavage, c'était d'abord une illusion historique comme Marc Bloch l'a bien montré — et ensuite une sottise, car les choses ne sont pas si simples que cela, comme l'enseigne "le vrai marxisme". J'ai salué ce "marxisme vivant" qui nous vient de Russie, et qui nous change du "marxisme fossile" des vieux guesdistes, ces catéchistes vivants et irréels. Mon sentiment est net. Dans dix ans, tout ce qui comptera en histoire sera "marxiste", d'un marxisme très souple, très large, peut-être hérétique (je n'en sais rien! et je ne me soucie pas de le savoir !) en tout cas, très compréhensif et contre lequel ni vous ni moi ne pouvons élever d'objections fondamentales. C'était assez émouvant à constater. » (Lettre du 29 mars 1936, Bloch et Febvre, 2003, p. 410).

Cette réévaluation du marxisme est favorisée par la politisation que connaît le monde universitaire français. À cette époque, l'assouplissement des contraintes politiques dans l'espace communiste accélère son ouverture vers le monde académique. Le PC se constitue alors en pôle d'attraction, même dans les milieux universitaires pourtant réputés réfractaires à toute forme militante et partisane d'engagement, mais néanmoins de plus en plus politisés, à partir de 1934. Cette attirance était autant politique que culturelle. Politique avec le combat pour la paix, la lutte contre le fascisme et le soutien aux républicains espagnols. Politique encore avec les luttes qui opposaient au Quartier latin les intellectuels de gauche à l'Action française, affaiblie certes par la condamnation pontificale de 1926, mais conservant tout de même une forte influence. Attrait culturel également du PC, qui se construit au milieu des années 1930 comme le garant du rationalisme philosophique né des Lumières, du rationalisme politique issu des traditions révolutionnaires françaises, et aussi du rationalisme savant opposé à l'anti-modernisme lettré.

La politisation du monde universitaire-scientifique contribue donc à légitimer la position que G. Friedmann entend occuper entre sciences sociales et communisme soviétique. Ses travaux sociologiques sur l'idée de progrès et la question des techniques présentaient, on l'a vu, un caractère hybride, en raison de leurs « adhérences souterraines » ${ }^{23}$ avec le discours communiste. Ils n'en furent pas moins reçus avec un vif intérêt par les directeurs des Annales, attentifs alors à la montée des fascismes et de l'antisémitisme (Müller, 2003). Ils apportaient, en effet, des éléments de réponse aux critiques qui se multipliaient dans l'entre-deux-guerres contre la science et le progrès technique et face auxquelles les fractions universitaires les plus militantes se mobilisaient : c'est

$\overline{23}$ Cette notion d'adhérence souterraine est reprise de P. Bourdieu (Bourdieu et Chartier, 2010, pp. 59). 
là une des principales raisons de la reconnaissance académique des écrits de G. Friedmann et plus largement du programme marxiste d'études du travail et des techniques auquel il contribua (avec A. Varagnac, C. Parain, A.-G. Haudricourt et d'autres) et qui fut, à partir de 1935, soutenu par l'équipe des Annales ${ }^{24}$.

\subsection{La réception académique de De la Sainte Russie à l'URSS}

La réception que connaît en 1938 l'ouvrage de G. Friedmann, De la Sainte Russie à l'URSS, témoigne de la reconnaissance académique dont il bénéficie désormais. Publié aux Éditions Gallimard, ce livre relève pourtant d'un genre particulier, mêlant prises de position politiques et enquête sociologique : G. Friedmann y propose un bilan de la situation soviétique, en centrant ses analyses sur les secteurs sociaux qu'il a lui-même observés lors de ses séjours en URSS (l'école, la formation professionnelle, l'organisation du travail, la vie intellectuelle et les questions morales). En fait, cette publication apparaît conforme au rôle politico-intellectuel qu'il a endossé depuis le milieu des années 1930 .

D'abord, la posture adoptée est explicitement celle d'un intermédiaire entre monde savant et monde communiste, associant «esprit critique» et «sympathie» à l'égard de l'expérience soviétique. G. Friedmann entend ainsi se démarquer des discours « alleluistes » et aussi des nombreux «témoignages où l'ignorance du pays, de son histoire, de sa population se double d'une incompréhension systématique de la Révolution » (Friedmann, 1938, pp. 13-19).

Le rôle de médiation scientifique entre l'URSS et la France est également clairement affiché. G. Friedmann se propose, en effet, de pallier les difficultés qu'ont connues « tous ceux qui dans un domaine quelconque de l'activité intellectuelle ont essayé de se renseigner avec précision sur ce qui se fait, se pense, se discute en URSS » (Friedmann, 1938, pp. 127). C'est dans cette perspective qu'il s'attache à présenter les enjeux et les modalités des discussions intellectuelles en URSS. Sont ainsi exposés dans l'ouvrage les débats philosophiques soviétiques, les discussions méthodologiques «contre la sociologie vulgaire » (son schématisme, son économisme, son mécanisme) ou encore la «querelle du formalisme » dans les domaines artistiques et littéraires. G. Friedmann décrit aussi les méthodes de discussion en vigueur en URSS, évoque «l'âpreté inouïe » des débats et la «dévotion» dont font l'objet les textes de Marx et de Lénine.

Enfin, en présentant son livre comme l'œuvre «d'un sociologue, intéressé avant tout par les problèmes psychologiques et moraux de [son] temps », G. Friedmann revendique un rôle de conseiller. Le regard distancié qu'il porte sur l'expérience soviétique le conduit à souligner certains problèmes, à indiquer « les erreurs commises » et à formuler un ensemble de recommandations. Tout l'ouvrage est traversé par ce ton critique qui se veut constructif.

Dans l'univers des sciences sociales durkheimiennes, la réception du livre de G. Friedmann sur l'URSS fut très positive. Les comptes rendus que font paraître L. Febvre dans les Annales ${ }^{25}$, M.

\footnotetext{
${ }^{24}$ En novembre 1935, un numéro spécial des Annales fut consacré à l'histoire des techniques. Y collaborèrent, entre autres, G. Friedmann, avec un article sur F.W. Taylor, et C. Parain, avec un article présentant les recherches menées, en URSS, par N. Vavilov sur l'origine des plantes cultivées. L. Febvre livra également dans ce numéro un compte rendu détaillé de l'ouvrage À la lumière du marxisme (Paris, ESI, 1935), publié par la commission scientifique du Cercle de la Russie neuve (dont C. Parain était, comme G. Friedmann, un membre actif).

${ }^{25}$ L. Febvre, «De la Sainte Russie à l'URSS», Annales HES, t. 10, 53, 30 septembre 1938, p. 442-445.
} 
Halbwachs dans les Annales sociologiques ${ }^{26}$ et C. Bouglé dans Marianne ${ }^{27}$ sont tous élogieux. M. Halbwachs parle d' " attachante étude économique, conduite avec une sympathie latente pour ce grand effort collectif, mais aussi avec une préoccupation remarquable d'objectivité, qui s'inspire de cette sympathie même». L. Febvre salue cet «effort vigoureux pour regarder les choses en face, sans parti pris de s'abuser volontairement ». Même C. Bouglé, qui pourtant s'était montré très critique vis-à-vis du marxisme et de la politique soviétique, conclut sa recension par un «bravo », et ajoute :

«Au bout du compte, nous nous trouvons en face d'un marxisme, ne disons pas édulcoré, mais enrichi et assoupli, prêt à tolérer, à accueillir, à rechercher des nuances d'explications qui eussent fait frémir d'horreur, en notre temps d'étudiant, un P. Lafargue. Le plus curieux, c'est qu'à cet enrichissement, à cet assouplissement intellectuel, la Russie elle-même, la Russie des Soviets collabore.»

\section{L'affaire Georges Friedmann : la fermeture de l'espace des possibles dans le monde communiste}

Au sein de l'univers durkheimien, G. Friedmann est donc parvenu à faire reconnaître sa position entre sciences sociales et communisme. Or, au moment même où ce nouveau rôle de l'intellectuel devient légitime dans les milieux universitaires, les logiques de fermeture, qui, avec la stalinisation, travaillent le PC, le rendent finalement caduque : c'est ce qu'on montrera en analysant la réception que connaît dans le monde communiste l'ouvrage De la Sainte Russie à l'URSS. Cette étude s'appuie sur le dossier qu'avait constitué, à la fin des années 1960, G. Friedmann, avec l'aide de Marc Ferro, en vue d'une réédition du livre ${ }^{28}$. Ce projet ne put aboutir, et seule la correspondance que G. Friedmann avait échangée à cette époque fut finalement publiée par Catherine MelnikDuhamel dans son mémoire de $\mathrm{DEA}^{29}$. La démarche de G. Friedmann témoigne, quoiqu'il en soit, de l'importance qu'il accordait à cet épisode. La réception très critique de son livre par les instances communistes marquera, on le sait, la fin de son engagement comme «compagnon de route » (Melnik-Duhamel, 1985 ; Racine, 1997). Les effets de cette « affaire » ne se limitèrent pas cependant à ces dimensions individuelles : c'est bien plus la définition du rôle des intellectuels de profession dans le monde communiste qui fut alors en jeu.

\subsection{De la Sainte Russie à l'URSS : une étude sociologique évaluée en fonction des règles et normes de l'univers communiste stalinisé}

Après la publication du livre de G. Friedmann en 1938, l'ensemble du dispositif de presse communiste est mobilisé en vue de définir et d'imposer la juste lecture que les militants doivent suivre. Des recensions de l'ouvrage paraissent non seulement dans la revue culturelle du PC (Commune), mais aussi dans les journaux et organes politiques (Cahiers du

\footnotetext{
26 M. Halbwachs, «Friedmann (Georges), De la Sainte Russie à l’URSS», Annales sociologiques, Série D, Sociologie économique, 1939, p. 112-114.

27 C. Bouglé, «Variations sur le marxisme », Marianne, 286, 13 avril 1938. Marianne était un hebdomadaire littéraire et politique, lancé en 1932 par Gaston Gallimard et dirigé par Emmanuel Berl jusqu'en 1937.

28 Ce dossier est conservé dans les archives privées de G. Friedmann.

29 Ce mémoire de DEA est consultable à la Bibliothèque de Science Po.
} 
Bolchevisme, L'Humanité, Russie d'Aujourd'hui) $)^{30}$. Cette diversité des lieux de publication atteste l'attention que porte au nouveau livre de G. Friedmann la direction communiste, en premier lieu Maurice Thorez, dont les archives conservent aujourd'hui un dossier sur cet épisode.

Autre indice de cette importance politique, le profil des auteurs des comptes rendus : tous se recrutent parmi les cadres thoréziens et leurs alliés intellectuels. Fernand Grenier est un représentant typique de la nouvelle élite ouvrière communiste (Pudal, 1989, pp. 240-248) ${ }^{31}$. Jean Bruhat, Paul Nizan et Georges Politzer comptent eux parmi ces intellectuels de formation qui obtiennent, à partir de 1932, des postes à responsabilité dans le PC et qui furent ainsi étroitement associés à la promotion de la direction thorézienne. Tous ont donc pour mission dans le PC de «prémunir l'élite ouvrière de la concurrence avec les intellectuels traditionnels » (Pudal, 1989, pp. 180-192). Et c'est bien ce rôle qu'ils joueront lors de la réception du livre de G. Friedmann, en centrant leurs critiques sur le ton «professoral» (J. Bruhat), «doctoral» (G. Politzer) ou «clérical» (P. Nizan) dont aurait fait preuve l'auteur de De la Sainte Russie à l'URSS. Plus grave encore est l'accusation de trotskisme que porte G. Politzer dans sa recension, publiée dans les Cahiers du bolchevisme, l'organe du Comité central, et dotée de ce fait d'une autorité politique particulière. G. Friedmann, dont le but aurait été « de faire l'apologie de Trotsky », y est assimilé à la catégorie du «traître » et de «l'ennemi de classe».

On a pu avancer des raisons personnelles pour expliquer le ton très virulent de G. Politzer, qui connaît G. Friedmann depuis leur participation commune au groupe Philosophies, à la fin des années 1920. En réalité, ce conflit tient, pour l'essentiel, aux transformations du monde communiste et aux positions que l'un et l'autre y occupent. Tous deux incarnent, en effet, deux types de carrières militantes possibles dans l'espace communiste des années 1930. Une trajectoire d'homme double, avec G. Friedmann, dont l'autorité politique dépend de la reconnaissance qu'il acquiert dans le monde intellectuel. Ce profil militant reste l'objet de suspicions, puisqu'il suppose d'accorder aux intellectuels traditionnels une certaine légitimité, ce qui pourrait par la suite leur permettre de contester la nouvelle élite ouvrière communiste. G. Politzer, en revanche, illustre une trajectoire de conversion. Il tend à devenir, au début des années 1930, un professionnel de la politique et, afin d'assurer et de consolider sa position dans la hiérarchie communiste, il doit faire siennes les nouvelles règles stalinisées du fonctionnement partisan. La recension du livre de G. Friedmann lui offre l'occasion de certifier sa fiabilité politique, la lutte anti-trotskiste servant alors, comme le soulignent Claude Pennetier et Bernard Pudal, de «rite d'intégration à la communauté des "fidèles" » et de «test de "l'esprit de parti"» (Pennetier et Pudal, 2006). Ainsi, le conflit qui éclate entre G. Friedmann et G. Politzer ne peut être réduit à des enjeux de personne. D'ailleurs, la transformation de ce conflit en véritable «affaire», suite aux efforts de mobilisation déployés par G. Friedmann, le montre.

\footnotetext{
${ }^{30}$ Les comptes rendus parus dans la presse communiste sont: P. Nizan, Compte rendu de De la Sainte Russie à l'URSS, Commune, mai 1938 ; G. Politzer, « À propos d'un livre sur l'URSS », Cahiers du Bolchevisme, mai-juin 1938 (Publication d'extraits de l'article dans L'Humanité); J. Bruhat, Compte rendu de De la Sainte Russie à l'URSS, L'Humanité (rubrique Doctrine et histoire), 21 avril 1938 ; F. Grenier, «Un livre de Georges Friedmann », Russie d'aujourd'hui (revue des Amis de l'Union soviétique), juillet 1938.

31 Bien que F. Grenier dispose d'un capital scolaire suffisant pour aspirer à une forme d'ascension sociale vers les classes moyennes, ce fils d'ouvrier reste attaché à son milieu familial : il adhère au PC en 1922 sur le mode de la fidélité à sa classe d'origine, et, en 1932, devient président des Amis de l'Union soviétique.
} 


\subsection{L'extension de la controverse et la mobilisation des intellectuels de profession}

Le 24 juin 1938, en effet, G. Friedmann écrit à M. Thorez, afin de dénoncer l'attaque dont il fait l'objet :

«Cher Maurice Thorez, Je viens seulement de prendre connaissance de l'article consacré par G. Politzer à mon livre sur l'URSS dans les Cahiers du Bolchevisme. Sans attendre la réponse appropriée qui lui sera faite, je tiens à vous dire ma profonde tristesse (et je crois pouvoir vous confier qu'elle est partagée par F. Jourdain, et d'autres amis dévoués du PC) de voir ma pensée, [...], mon texte déformés d'une manière aussi tendancieuse : et j'ajoute haineuse, l'accent de haine ayant frappé tous ceux qui m'ont parlé de cet article.

Je n'analyserai pas ici les artifices odieux par lesquels cet article cherche à présenter comme un trotskiste camouflé un homme qui n'a pas une seule liaison trotskiste. Au reste, vous avez lu le livre avec une attention critique et vous pouvez juger. Je ne suis pas seul à penser que de tels articles desservent la cause de l'Union soviétique et du PCF.

Bien fraternellement à vous. $»^{32}$

Afficher les soutiens dont il bénéficie parmi les intellectuels sympathisants dotés d'une certaine autorité dans le mouvement communiste, tel est le principal procédé utilisé par G. Friedmann pour accomplir le travail de «dé-singularisation », nécessaire au succès de sa dénonciation (Boltanski et al., 1984). Dès son premier échange avec M. Thorez, G. Friedmann fait valoir ses relations avec Francis Jourdain, le préfacier de son livre, et «d'autres amis dévoués du PC ». Ce procédé prendra rapidement une autre envergure, lorsque des intellectuels « sympathisants » reconnus dans le monde communiste (Francis Jourdain et Romain Rolland ${ }^{33}$, puis Henri Wallon, Paul Langevin, Jean Luc, René Maublanc ${ }^{34}$ et d'autres) prendront position, en envoyant à M. Thorez des lettres de soutien.

La correspondance qu'échange G. Friedmann avec F. Jourdain et R. Rolland durant l'été 1938 le montre. Toutes ces lettres relèvent bien d'une riposte réfléchie. Elles reposent sur un travail de mobilisation au cours duquel G. Friedmann s'applique à faire de son cas un problème politique général, celui de l'autonomie des intellectuels dans le monde communiste. Est ainsi soulevée, dès la fin des années 1930, la question des rapports que les intellectuels de profession peuvent et doivent entretenir avec la direction communiste. C'est cet enjeu qui sera au centre des négociations à l'été 1938.

\subsection{La négociation et le dénouement avorté de l'affaire}

Début août, notamment, M. Thorez rendra visite à R. Rolland en résidence à Vézelay. Dans ce cadre de discussion qui relève autant de l'amitié que de la négociation politique, M. Thorez pourra exposer ses propres critiques et par là expliciter les règles qui régissent les rapports des intellectuels au PC : non-intervention dans les affaires politiques internes, même sur un mode intellectualisé (celui, par exemple, de l'analyse sociologique), droit de contrôle et de critique de la direction

\footnotetext{
32 Lettre de G. Friedmann à M. Thorez, 24 juin 1938. Fonds M. Thorez, Carton 62AP/53 (Archives nationales).

${ }^{33}$ F. Jourdain et R. Rolland écrivent à M. Thorez dans le courant du mois de juillet. Ces deux intellectuels sympathisants, dont la proximité avec M. Thorez est forte, réaffirment dans leur lettre les intentions positives de G. Friedmann à l'égard de la cause communiste. Lettre conservée dans le fonds M. Thorez, Carton 62AP/53 (Archives nationales).

34 Voir leurs notices biographiques dans (Pennetier, 1997).
} 
politique sur leurs productions (avant et après diffusion), interdiction de toute organisation autonome d'intellectuels susceptible d'exercer une pression sur les instances dirigeantes ${ }^{35}$.

De cette rencontre informelle entre $\mathrm{M}$. Thorez et R. Rolland, émerge un compromis que G. Friedmann approuvera par la suite ${ }^{36}$. Ce dernier est autorisé à publier dans les Cahiers du bolchevisme la réponse qu'il a rédigée à $\mathrm{G}$. Politzer à deux conditions : d'une part, la suppression dans son texte de toute référence aux amis intellectuels sur l'autorité desquels il s'est appuyé — cette mesure vise à rendre invisible la mobilisation des intellectuels que l'affaire a entraînée -, d'autre part, la publication d'une «mise au point» de M. Thorez à la suite de son article — cette clause a pour objectif de renforcer l'autorité du secrétaire général du PC, en faisant de lui l'arbitre des relations entre intellectuels dans le monde communiste.

À l'automne 1938, l'affaire est donc sur le point de se régler. Pourtant la réponse de G. Friedmann ne sera jamais publiée, ni la mise au point de M. Thorez. Tandis qu'au terme de cet épisode, G. Politzer conforte sa position au sein de la hiérarchie partisane, la situation de G. Friedmann dans le PC demeure ambiguë. Sans être démis officiellement de ses responsabilités, il est néanmoins marginalisé au sein du monde communiste et n'est pas autorisé à répondre dans la presse communiste aux attaques de G. Politzer.

En fait, dès l'automne 1938, la mobilisation construite par G. Friedmann avait commencé à s'effriter. Les menaces croissantes de guerre créaient alors un climat d'apesanteur politique et incitaient les intellectuels à suspendre leurs désaccords avec la direction du PC. «L'explication très sincère » qu'appelaient de leurs vœux G. Friedmann, H. Wallon, F. Jourdain et d'autres, avec les responsables communistes n'eut de ce fait jamais lieu. La question, qu'ils avaient réussi à mettre en débat, du degré d'autonomie dans l'entreprise culturelle communiste ne fut pas examinée.

C'est donc dans une position politique très incertaine que G. Friedmann reçoit la nouvelle du Pacte signé entre l'URSS et l'Allemagne le 23 août 1939. Cet événement rend alors possible le délitement de son identification partisane, déjà mise à mal en 1938, voire en $1936^{37}$. Il accélère le processus de distanciation à l'égard du PC, processus qui ne pouvait auparavant aboutir en raison de l'image que détenait le communisme soviétique dans la lutte antifasciste. Le Journal de guerre de G. Friedmann se lit ainsi comme une réévaluation critique de la politique communiste et de son propre passé militant. Mobilisé à Laon, il écrit, par exemple, le 25 septembre 1939:

«Avoir eu raison et être seul, craint, livré aux injures et abandonné même par des amis, de braves gens, comme un "lépreux intellectuel": condition de celui qui, sans renier ses

\footnotetext{
35 Voir la lettre de R. Rolland à G. Friedmann du 29 août 1938 (Lettre reproduite dans C. Melnik-Duhamel, 1985, pp. 218-219).

36 Si ce compromis se construit au cours de rencontres informelles et d'échanges épistolaires, sa finalisation prend un tour plus officiel à l'automne 1938. G. Friedmann rencontrera alors un cadre communiste, devant lequel il pourra énoncer ses propres conditions. Il demandera notamment à ce que sa réponse soit publiée entièrement dans les Cahiers du bolchevisme et sous forme d'extraits dans L'Humanité, comme il en a été pour la recension de G. Politzer. Surtout, il soulignera combien l'intervention de M. Thorez conditionne son maintien aux côtés du PC: «Dans les circonstances actuelles, où je me sens plus que jamais d'accord avec le Parti communiste dans sa lutte contre l'hitlérisme et la guerre, j'espère que la mise au point sera rédigée de manière à me permettre de conserver, aux côtés du Parti, la place d'ami, de combattant et aussi la possibilité morale de l'occuper» (Note remise au secrétariat du Parti communiste, comme suite aux propositions faites par M. Thorez pour le règlement de l'incident Politzer. Fonds M. Thorez, Carton 62AP/53, Archives nationales).

37 Dans de multiples récits autobiographiques, G. Friedmann fait remonter à 1936 ses premiers doutes sur l'URSS et le communisme soviétique. De fait, les rapports rédigés par les responsables du VOKS lors de son séjour en URSS de 1936 rapportent des propos assez critiques de G. Friedmann, en particulier au sujet des procès de Moscou (Coeuré et Mazuy, 2012, document 113).
} 
espoirs en l'URSS, avait sonné la cloche d'alarme. Il est dur de la soutenir, à la longue. J'ai connu cette épreuve pendant plus d'un an. Toute épreuve de ce genre est bonne puisqu'elle fortifie l'esprit et le caractère.

Si mes journées n'étaient pas rongées par les tâches de l'HC, si je n'étais pas trop las, le soir, lorsque je regagne ma chambre, je m'efforcerais de mettre au point une sorte d'examen dont je sens l'utilité pour beaucoup de gens que je connais.

Comment cette déchéance de la raison s'est-elle produite progressivement, depuis quelques années, parmi des hommes d'esprit et de cœur?» (Friedmann, 1987, pp. 56-57).

\section{Conclusion}

Dans le monde communiste stalinisé, les «affaires » ont constitué « un mode de régulation du corps militant », permettant d'assurer « la préservation de l'unité organique du parti » (Boulland, 2011, chap. 2). En 1938, la condamnation du livre de G. Friedmann, De la Sainte Russie à l'URSS, répond à cette visée. Elle entraîne la marginalisation d'un militant jugé gênant et, en raison de sa valeur exemplaire et pédagogique, elle permet la disqualification de certaines manières d'être intellectuel dans le mouvement communiste. De fait, la position politico-intellectuelle que G. Friedmann était parvenu à faire exister à la jonction des sciences sociales et du communisme soviétique s'avérait intenable à la fin des années 1930.

D'abord, la répression politique qui avait violemment frappé le monde intellectuel soviétique lors des grandes purges staliniennes de 1937-1938 avait rendu caduque la fonction de médiation scientifique que G. Friedmann exerçait entre l'URSS et la France. Même le VOKS, qui avait été une de ses principales voies d'entrée dans le monde scientifique soviétique, était alors décimée (David-Fox, 2003). À la fin des années 1930, les circuits de diffusion franco-soviétiques auxquels G. Friedmann et les intellectuels sympathisants avaient contribué étaient donc complètement désorganisés. D'ailleurs, la plupart des travaux soviétiques qui avaient nourri leurs réflexions, ceux notamment de l'école psychotechnique d'I.N.Spielrein, de l'historien des sciences B. Hessen, du botaniste N. Vavilov ou encore d'I. Luppol, n'étaient plus légitimes dans l'univers communiste.

En outre, le rôle d'entrepreneur culturel que jouait G. Friedmann dans le monde communiste était soumis à des tensions croissantes, la présence des intellectuels étant désormais perçue par la direction thorézienne comme une menace trotskiste. Une telle vision était révélatrice des contradictions sous-jacentes à la politique communiste mise en œuvre vis-à-vis des intellectuels de profession. Si la caution symbolique et les compétences professionnelles que ces derniers apportaient au projet communiste confortaient le travail de légitimation culturelle et politique de la nouvelle élite thorézienne, elles pouvaient en même temps servir d'appui à une contestation interne au monde communiste. Au printemps 1938, la direction communiste thorézienne se saisira donc de la publication du livre de G. Friedmann, De la Sainte Russie à l'URSS, pour engager la renégociation de ses rapports avec les milieux intellectuels philosoviétiques. Quelques mois plus tard, en 1939, le lancement de la revue La Pensée aboutira à la mise en place d'un nouveau mode de gestion des intellectuels, adapté au monde communiste stalinisé. C'est désormais dans le cadre localisé et politiquement contrôlé de la revue que les tensions avec les intellectuels devront se régler ${ }^{38}$ ou, si nécessaire, sur le mode de «l'affaire».

\footnotetext{
$\overline{38}$ Sur les revues et la gestion des intellectuels dans le monde communiste, cf. (Matonti, 2005).
} 
Dès la fin des années 1930 donc, la stalinisation et les repositionnements politico-intellectuels qu'elle entraîne annoncent l'éclatement du programme de recherches marxistes, né de la rencontre entre l'univers des sciences humaines françaises et le monde communiste. La sociologie que G. Friedmann s'efforcera de promouvoir après 1945 (Borzeix et Rot, 2010; Tanguy, 2011) pourrait néanmoins être lue comme un des prolongements possibles, mais coupé désormais de son point d'ancrage politique initial, de ce programme qui faisait du travail le lien social par excellence et qui proposait, à partir de ce postulat, une science sociale unifiée. Il resterait alors à examiner les traces de cette filiation dans la sociologie du travail d'après la Seconde Guerre mondiale, que ce soit dans la définition même de son objet, dans son ouverture disciplinaire vers la psychologie et l'histoire sociale des Annales, dans ses liens avec le monde de la pratique, ou encore dans les dialogues qu'elle a poursuivis avec les marxismes.

\section{Déclaration d'intérêts}

L'auteur déclare ne pas avoir de conflits d'intérêts en relation avec cet article.

\section{Références}

Bloch, M., Febvre, L., 1994-2003. Correspondance. Édition établie, présentée et annotée par B. Müller., Gallimard, Paris. Boltanski, L., Darré, Y., Schiltz, M.A., 1984. La Dénonciation. Actes de la recherche en sciences sociales 51, 3-40.

Borzeix, A., Rot, G., 2010. Genèse d'une discipline, naissance d'une revue. Sociologie du travail. Presses universitaires de Paris Ouest, Paris.

Bouju, M.C., 2010. Lire en communiste, les maisons d'édition du Parti communiste français, 1920-1968. PUR, Rennes.

Boulland, P., 2011. Acteurs et pratiques de l'encadrement communiste à travers l'exemple des fédérations de banlieue parisienne (1944-1974). Thèse de l'Université Paris 1, Paris.

Bourdieu, P., Chartier, R., 2010. Le sociologue et l'historien. Agone, Marseille.

Charle, C., 1998. Paris, fin de siècle. Culture et politique. Seuil, Paris.

Coeuré, S., Mazuy, R. (Eds.), 2012. Cousu de fil rouge (Voyages des intellectuels français en Union soviétique). CNRS Éditions, Paris.

Coeuré, S., 1999. La Grande lueur à l'est. Les Français et l'Union soviétique, 1917-1939. Seuil, Paris.

Coeuré, S., 2012. Introduction : retour sur le «retour d'URSS ». Dans les coulisses d'un mythe politique et littéraire. In: Coeuré, S., Mazuy, R. (Eds.), Cousu de fil rouge (Voyages des intellectuels français en Union soviétique). CNRS Éditions, Paris, pp. 9-34.

Coeuré, S., 1995. Les centres de documentation sociale, 1920-1940. In: Beausoleil, J., Ory, P. (Eds.), Albert Kahn, 1860-1940 : réalités d'une utopie. Musée A. Kahn/Département des Hauts de Seine, Boulogne, pp. 201-208.

David-Fox, M., 2012. Showcasing the Great Experiment. Cultural Diplomacy and Western Visitors to the Soviet Union, 1921-1941. Oxford University Press, Oxford, New York.

David-Fox, M., 2003. Stalinist Westernizer? Aleksandr Arosev's Litera rand Political Depictions of Europe. Slavic Review 62 (4), 733-759.

Dreyfus, M., 1997. Célestin Bouglé. In: Pennetier, C. (Ed.), Dictionnaire biographique du mouvement ouvrier. Le Maitron. Éditions de l'Atelier, Paris.

Dumoulin, O., 2003. Le rôle social de l'historien. De la chaire au prétoire. Albin Michel, Paris.

Durkheim, E., 1987 [1970]. La science sociale et l'action. Puf, Paris.

Fayet, J.F., 2003. La Société pour les échanges culturels entre l'URSS et l'étranger (VOKS). Relations internationales $115,411-423$.

Filoni, M., 2010 [2008]. Le philosophe du dimanche. La vie et la pensée d'Alexandre Kojève. Gallimard, Paris.

Fleck, L., 2005 [1935]. Genèse et développement d'un fait scientifique. Les Belles lettres, Paris.

Friedmann, G., 1938. De la Sainte Russie à l'URSS. Gallimard, Paris.

Friedmann, G., 1987. Journal de guerre, 1939-1940. Gallimard, Paris.

Friedmann, G., 1935. Machine et humanisme. Thèses présentées au Congrès international des écrivains pour la défense de la culture. Europe, $n^{\circ}$ juillet, 437-444. 
Gouarné, I. 2010. Philosoviétisme et rationalisme moderne. L'introduction du marxisme dans les sciences humaines en France (1920-1939). Thèse Université de Nantes, Nantes.

Grémion, P., Piotet, F. (Eds.), 2004. Georges Friedmann, un sociologue dans le siècle, 1902-1977. CNRS Éditions, Paris.

Heilbron, J., 1985. Les métamorphoses du durkheimisme, 1920-1940. Revue française de sociologie 26 (2), $203-237$.

Humphreys, J.M., 1999. Durkheimian Sociology and 20th-century: The Case of Célestin Bouglé. History of the Human Sciences 12 (3), 117-138.

Israël, S., Mochon, J.P., 1994. Les normaliens et la politique. In: Sirinelli, J.F. (Ed.), L’École normale supérieure. Le livre du bicentenaire. Puf, Paris, pp. 191-214.

Jarczyk, G., Labarrière, P.J., 1990. Cent cinquante ans de « réception » hégélienne en France. Genèses 2, 109-130.

Lacroix, B., 1978. À propos des rapports entre Durkheim et Marx : de l'analyse de texte à l'analyse sociologique. In: Université de Clermont-Ferrand, Faculté de droit et de science politique, Études offertes au Professeur Emérentienne de Lagrange. Librairie générale de droit et de jurisprudence, Paris, pp. 329-50.

Logue, W., 1979. Sociologie et politique : le libéralisme de Célestin Bouglé. Revue française de sociologie 20 (1), $141-161$.

Marcel, J.C., 2001. Le durkheimisme dans l'entre-deux-guerres. Puf, Paris.

Matonti, F., 2005. Intellectuels communistes. Essai sur l'obéissance politique. La Nouvelle critique (1967-1980). La Découverte, Paris.

Matonti, F., 2004 [2000]. Les intellectuels et le Parti. In: Dreyfus, M. et al. (Eds.). Le Siècle des communismes. Seuil, Paris, pp. 603-32.

Mazuy, R., 2004. Des voyages aux doutes: Georges Friedmann en URSS. In: Grémion, P., Piotet, F. (Eds.), Georges Friedmann, un sociologue dans le siècle, 1902-1977. CNRS Éditions, Paris, pp. 21-28.

Mazuy, R., 2002. Croire plutôt que voir? Voyages en Russie soviétique (1919-1939). Odile Jacob, Paris.

Melnik-Duhamel, C., 1985. L'affaire Georges Friedmann : à propos de la publication de « De la Sainte Russie à l'URSS ». Mémoire de DEA de l'IEP de Paris, Paris.

Müller, B., 2003. Introduction à Bloch, M., Febvre, L., Correspondance, 1934-1937. Fayard, Paris.

Pennetier, C., Pudal, B., 2006. La peur de l'autre: vigilance anti-trotskiste et travail sur soi. In: Studer, B., Haumann, H. (Eds.), Stalinistische Subjekte/Stalinist Subjects/Sujets staliniens. Individuum und System in der Sowjetunion und der Komintern, 1923-1953. Chronos, Zürich, pp. 253-275.

Pennetier, Cl. (Ed.), 1997. Dictionnaire biographique du mouvement ouvrier. Le Maitron. Éditions de l'Atelier, Paris.

Pollak, M., 1988. La place de Max Weber dans le champ intellectuel français. Droit et société 9, 195-210.

Prochasson, C., 1999. Entre science et action sociale : le réseau Albert Thomas et le socialisme normalien, 1900-1914. In: Topalov, C. (Ed.), Laboratoires du nouveau siècle. La nébuleuse réformatrice et ses réseaux en France (1880-1914). Éditions de l'EHESS, Paris, pp. 141-158.

Pudal, B., 2002. Récits édifiants du mythe prolétarien et réalisme socialiste en France (1934-1937). Sociétés et représentations 15, 77-96.

Pudal, B., 2004. Pour une histoire de l' «intellectuel collectif» communiste avec et contre les enseignants (1920-2000). In: Girault, J. (Ed.), Les enseignants dans la société française au $\mathrm{xx}^{\mathrm{e}}$ siècle. Publications de la Sorbonne, Paris, pp. $181-212$.

Pudal, B., 1989. Prendre parti. Pour une sociologie historique du PCF. Presses de la FNSP, Paris.

Racine, N., 1997. Georges Friedmann. In: Pennetier, C. (Ed.), Dictionnaire biographique du mouvement ouvrier, Le Maitron. Éditions de l'Atelier, Paris.

Racine, N., 1967. Une revue d'intellectuels communistes dans les années 1920: Clarté (1921-1928). Revue française de science politique 3, 484-519.

Rey, M.P., 2002. Le Dilemme russe. La Russie et l'Europe occidentale d'Ivan le Terrible à Boris Eltsine. Flammarion, Paris.

Ribeill, G., 1999. Approches critiques du travail industriel entre les deux guerres: la place de Georges Friedmann. Sociologie du travail 41 (1), 23-39.

Ribeill, G., 1984. Les organisations du mouvement ouvrier en France face à la rationalisation (1926-1932). In: de Montmollin, M., Pastré, O. (Eds.), Le Taylorisme. La Découverte, Paris, pp. 127-140.

Rot, G., Vatin, F., 2004. Les avatars du «travail à la chaîne » dans l'œuvre de Georges Friedmann (1931-1966). Genèses $57,23-40$.

Stern, L., 2007. Western Intellectuals and the Soviet Union, 1920-1940. From Red Square to the Left Bank. Routlegde, Londres \& New York.

Tanguy, L., 2011. La sociologie du travail en France. Enquête sur le travail des sociologues, 1950-1990. La Découverte, Paris.

Topalov, C., 2001. Des livres et des enquêtes : pour un historicisme réflexif. In: Lepetit, B., Topalov, C. (Eds.), La ville des sciences sociales. Belin, Paris, pp. 307-313. 
Tournès, L., 2008. La fondation Rockefeller et la construction d'une politique des sciences sociales en France (1918-1940). Annales HSS 63 (6), 1371-1402.

Trebitsch, M., 1987. Les mésaventures du groupe Philosophies, 1924-1933. La revue des revues 3, 6-11.

Vatin, F., 2004. Machinisme, marxisme, humanisme : Georges Friedmann avant et après-guerre. Sociologie du travail 46 (2), 205-223.

Verret, M., 1972. Halbwachs ou le deuxième âge du durkheimisme. Cahiers internationaux de sociologie 53, 311-336. 